\title{
Sexually transmitted infections in Iran: A literature review
}

\author{
Ghorashi Z, PhD
}

- Assistant Prof., Dept. of Midwifery, Faculty of Nursing Midwifery, Rafsanjan University of Medical Sciences, Rafsanjan, Iran.

\begin{abstract}
Received: April 2016, Accepted: October 2016

Background: The global burden of sexually transmitted infections (STIs) is noticeable and important. However, most STIs are curable and all of them are preventable. In this article, we reviewed and evaluated STI prevalence, current services, existing programs, and the challenges of control and management them in Iran.
\end{abstract}

Materials and Methods: The author searched literature published from 2005 to 2015 in PubMed, MEDLINE, Google, Embase, and Cochran library databases, and by UNAIDS, , the World Health Organization (WHO), and the Health Ministry of Iran. A variety of Medical Subject Headings $(\mathrm{MeSH})$ terms were used to access the literature.

Results: The present review revealed that the prevalence of contamination with hepatitis B virus (HBV) in Iran has decreased dramatically in recent years. The prevalence of chlamydia, gonorrhea, and syphilis in the Iranian population did not exceed the estimated prevalence of these infections in the Eastern Mediterranean Region. Although the condition of HIV epidemy is concentrated in Iran, its prevalence in the general population, based on literature, is extremely low. Centers of behavioral disease counseling, women centers, and positive clubs are three available centers in Iran helping in STI/AIDS prevalence reduction. The changing trend of HIV transmission pattern and lack of youth friendly services are the two main challenges of STI/AIDS prevention in Iran.

Conclusions: The overall prevalence of STIs and HIV/AIDS is low in Iran. However, neglecting youth sexual activity and premarital sexual relationships could result in the failure of the STIs and HIV/AIDS prevention programs.

Keyword: Sexually Transmitted Infections, Iran, Review

\section{Introduction}

Sexually transmitted infections (STI) consist of more than 30 viral, bacterial, and parasitic infections which are transmitted through person to person sexual contact including vaginal, oral, and anal sex. Some of these infections may be transmitted from mother to fetus, specially syphilis and HIV (1).

The global burden of disease caused by STIs is noticeable and important. More than one million people acquire an STI every day. Chlamydia, gonorrhoea, syphilis, and trichomoniasis are acquired by an estimated 500 million people each year worldwide. Hundreds of millions of people have the virus that causes genital herpes simplex virus type 2
(HSV2) and human papilomavirus (HPV) infection globally (2).

Most STIs are curable and all of them are preventable; thus, attention to the prevention and treatment of these infections would greatly impact public health. Although, the incidence and prevalence of STIs is low in the Eastern Mediterranean Region of the World Health Organization (WHO), acknowledgement of the situation is necessary (3).

The aim of this study was to review the prevalence of STIs in Iran, current services,

\footnotetext{
* Corresponding author: Zohreh Ghorashi, Dept. of Midwifery, faculty of Nursing Midwifery, Rafsanjan University of Medical Sciences, Rafsanjan, Iran.

E-mail: zghorashi@yahoo.com
} 
existing programs, and the challenges regard these issues. In this paper, rather than a systematic review, a simple review and evaluation was performed.

\section{Literature Search}

For the purpose of this paper, on August 2016 the author searched literature published from 2005 to 2015 in PubMed, MEDLINE, Google, Embase, and Cochrane library databases, and by UNAIDS, the WHO, and the Health Ministry of Iran. A variety of Medical Subject Headings (MeSH) terms were used to access the literature. The MeSH terms used to search in English and Persian were hepatitis B, Iran, HIV, AIDS, STIs, HBV, syphilis, chlamydia, and gonorrhea.

Prevalence of STIs in Iran: The most important and prevalent STIs in Iran, which are HBV, HSV2, chlamydia, gonorrhea, and syphilis, have been addressed in this section.

Hepatitis B Virus: Most studies in Iran regarding the prevalence of $\mathrm{HBV}$ have been conducted on blood donors. In a study on 3700 Southeastern Iranian blood donors, HBV-DNA was detected in $16.1 \%$ of blood samples (4). Moreover, the prevalence of HBV among 128198 blood donors from Golestan Province in Northern Iran have been studied retrospectively (5). The results revealed that the prevalence of $\mathrm{HBV}$ in 2006, 2007, and 2008 was $1.25 \%, 0.95 \%$, and $0.77 \%$, respectively (5). In another study on blood donors referred to Tehran Blood Transfusion Center (Tehran, Iran) from 2008 to 2013, the prevalence of $\mathrm{HBV}$ decreased dramatically during the study period. In this study, 1796090 blood donors were studied and the prevalence of HBV during the 6 years of the study decreased from 423 to 153 per 105 donors (6). Hepatitis B surface antigen (HBsAg) screening test is one of the laboratory tests performed on pregnant women in Iran. In a national study among 5261 pregnant women, HBsAg was positive in $1.2 \%$ of samples in 2011 (7). This prevalence was slightly higher $(1.56 \%)$ in pregnant women in the city of Kashan (Iran) in
2012 (8). In the same year, the prevalence of positive HBsAg among marriage candidates in the Southeast of Iran was $1.1 \%$ (9). In the general population in Northern Iran, the prevalence rate of positive $\mathrm{HBsAg}$ was $0.9 \%$ in 2008-2011 (10).

Although HBV is more prevalent in high risk groups, for example $34.7 \%$ in homeless men in Tehran (11), the overall prevalence of HBV among blood donors in Iran has decreased dramatically in recent years (12). This could be attributed to suitable measures taken by the Iranian Blood Transfusion Organization to decrease the transmission risks, and the vaccination of high risk groups and public vaccinations in recent years by the Health Ministry of Iran (13).

Chlamydia: Most of the studies on the prevalence of chlamydia in Iran have been undertaken on high risk groups or patients with signs and symptoms of STIs. These studies reported a prevalence of $9.3 \%$ to $34.71 \%$ for chlamydia infection (14-21). A study on 196 pregnant women attending maternal hospitals in Central Iran revealed a prevalence of $14.79 \%$ for chlamydia infection (22).

The global estimate for the prevalence of chlamydia among women in 2005 reported by the WHO was $3.53 \%$ (23). This report has estimated the prevalence of chlamydia infection in the Eastern Mediterranean Region as $2.15 \%$ (23). Similarly, a study on 255 married women aged 17-35 years, with or without symptoms, referring to the obstetrics and gynecology clinics in Central Iran, revealed a chlamydia infection prevalence of $2.4 \%$ (17). However, this rate was higher $(12.6 \%)$ in a study in Tehran in $2003(17,24)$. It seems that chlamydia infection prevalence in Iran is dramatically high, and in some areas, its prevalence exceeds the Eastern Mediterranean Region prevalence.

Gonorrhea: It seems that the prevalence of gonorrhea infection among Iranians is not high and does not exceed the prevalence estimated for the Eastern Mediterranean Region in 2005 by the WHO $(0.79 \%)$. 
A study on 500 female prisoners in Tehran Province and Alborz Province (Iran) revealed no case of gonorrhea infection (25). The prevalence of gonorrhea in pregnant women and in sex worker women was reported as $1.18 \%$ and $4.7 \%$, respectively, in two different studies $(26,27)$.

Syphilis: Syphilis in Iran is not a grave problem of the health system and maternal child health. Indeed, the prevalence of syphilis among Iranian blood donors has not been exceeded 10.5 per 100000 donations in relative studies and had a sharp decreasing trend in $2009(6,28,29)$. The prevalence of syphilis in high risk groups such as street children, female sex workers and prisoners, and homeless men have been reported from 0 to a maximum of $7.2 \%$ in different studies in different provinces $(11,19,26,30,31)$.

HSV2: Herpes simplex virus type 2 (HSV2) has not been rare in women attending the gynecologic clinics in Iran in recent years. In two different studies on female sex workers, the prevalence of HSV2 was reported as $9.7 \%$ and $18 \%(26,30)$.

HIV/AIDS: Although the condition of HIV epidemic is concentrated in Iran, its prevalence in the general population is extremely low (11, 12, 19, 28-33). The condition of Epidemic concentration is covering injecting drug users with prevalence of $12.3 \%-26.6 \%$ in Tehran and $18.8 \%-15.2 \%$ throughout the country (3436). According to the latest report, the frequency of HIV is 29414 cases, and AIDS is 6990 cases, 6202 cases of which died from the disease (37).

The incidence rate of HIV in Iran rose dramatically from 1995 to 2004, and since then, it has had a downward trend. In 2015, 2000 new cases of HIV infection were diagnosed nationwide (38). The number of HIV infected individuals in Iran has been rising regularly from 2001 to 2013 with a gentle growth (39). The prevalence of HIV infection among 15-49 year-old individuals has been estimated as $0.1 \%$ (39). However, only $14 \%$ of infected pregnant women received antiretroviral drugs to prevent maternal to child transmission in Iran in 2013 (40). Of all the infected cases, 450 cases are children who have acquired their infection from their mothers (38). The main way of transmission in Iran has been the sharing of syringes among injecting drug users; however, the contribution of sexual transmission has been rising in recent years. This rise is known as the third surge of AIDS. Fortunately, HIV transmission through blood transfusion has been eliminated in Iran (41). In addition, the percentage of infected women has been increasing recently (42).

\section{Available Services and Programs}

There are three types of centers offering services for reducing the probability of HIV transmission in Iran.

- Centers of behavioral disease counseling: These centers' services are under surveillance of universities of medical sciences all around Iran. The main services include education, counseling, and free and private HIV detection tests, drug use harm reduction via offering syringes, needles, and condoms. Phone counseling is also possible via these centers.

- Women centers: These centers have been established since 2006 and offer sexual reproductive health services and harm reduction services to high risk women. The health care providers in these centers are also women. A wide range of education, counseling, and free instruments are offered in these special centers.

- Positive clubs:Positive prevention is the main aim of these clubs and they offer a wide range of services for HIV infected individuals. Quality of life (QOL) promotion, psychosocial support, and health care are presented in these centers.

In Iran, retroviral drugs and all required services are offered free to HIV infected individuals (42). Generally, in Iran, ten 
strategies have been established for HIV/AIDS national prevention (43).

\section{STIs Prevention Challenges in Iran}

The overall prevalence of STIs and HIV/AIDS is low in Iran. This could be due to the Iranian religious and traditional culture of abstinence from premarital and extramarital sexual interactions. This culture could be changing dramatically now. However, the prevention program in Iran is faced with two main challenges. The first one is the changing trend of HIV transmission pattern from needle sharing pattern to sexual contact pattern that is known as the third surge of AIDS. It seems that sexual transmission prevention would be more problematic, especially because of the dramatic change in the youth sexual activity pattern and culture in Iran in recent years (44). The second challenge is the lack of youth friendly services in the Iranian health care program. The primary health care services have offered acceptable services in recent decades in Iran. However, launching youth friendly services as centers that could offer sexual reproductive health services to adolescents and young people has faced political and cultural resistance. Due to these two challenges, the STIs prevention programs may be faced with more difficulties in subsequent decades.

One other challenge is the HIV infection stigma in the Iranian culture. This stigma is a deterrent for the detection of the infection and provision of care measures that in most cases are provided freely by health systems.

\section{Conclusion}

Neglecting youth sexual activity and premarital sexual relationships could result in the failure of the STIs and HIV/AIDS prevention programs. Sexual reproductive education and counseling for adolescents and youth is an essential and urgent demand of the Iranian prevention programs. Further research on HIV transmission is also essential.
Conflict of interest: None declared

\section{Reference}

1. Swiss. World Health Organization. Sexually transmitted infections (STls). World Health Organization. World Health Organization: Media centre, Fact sheets; 2016.

2. Swiss.World Health Organization. Sexually transmitted infections(STIs). World Health Organization: Media centre, Fact sheets; 2013.

3. Toskin I. Epidemiology of STIs: factors, numbers and surveillance. Paper presented at: Training Course in Sexual and Reproductive Health Research, Foundation for Medical Education and Research; 2015 Jul 24; Geneva, Swiss.

4. Arababadi MK, Pourfathollah A, Jafarzadeh A, Hassanshahi G, Rezvani ME. Association of exon 9 but not intron 8 VDR polymorphisms with occult HBV infection in south-eastern Iranian patients. J Gastroenterol Hepatol 2010; 25(1):90-3.

5. Bani Aghil SS, Abbasi S, Arab M, Sayedin MS. The prevalence of HIV, HBV, HCV in blood donors in Golestan province, (20062008). Medical Labratory Journal 2009; 3(2):15.

6. Keshvari M, Sharafi H, Alavian SM, Mehrabadi H, Zolfaghari S. Prevalence and trends of transfusion-transmitted infections among blood donors in Tehran, Iran from 2008 to 2013. Transfus Apher Sci 2015; 53(1):3847.

7. Shoghli A, Nabavi SM, Alavian SM, Kolifarhood G, Goya MM, Namazi R, et al. Hepatitis B surface antigen prevalence in pregnant women: A cross-sectional survey in Iran. Int J Prev Med 2014; 5(Suppl 3):S213-8.

8. Afzali H, Momen Heravi M, Moravveji SA, Poorrahnama M. Prevalence of hepatitis B surface antigen in pregnant women in beheshti hospital of Kashan, Isfahan. Iran Red Crescent Med J 2015; 17(7):e20598.

9. Hayatbakhsh MM, Darvish Moghaddam S, Zahedi MJ, Shafiei M, Khalily Zade M, Assare M. Seroprevalence of hepatitis B before marriage: a study on marriage candidates in the Southeast of Iran; is it worthy of consideration? Arch Iran Med 2015; 18(1):34-8.

10. Keyvani H, Sohrabi M, Zamani F, Poustchi H, Ashrafi H, Saeedian F, et al. A population based study on hepatitis B virus in northern iran, amol. Hepat Mon 2014; 14(8):e20540.

11. Vahdani P, Hosseini-Moghaddam SM, Family A, Moheb-Dezfouli R. Prevalence of HBV, 
HCV, HIV and syphilis among homeless subjects older than fifteen years in Tehran. Arch Iran Med 2009; 12(5):483-7.

12. Mohammadali F, Pourfathollah AA. Changes in frequency of HBV, HCV, HIV and syphilis infections among blood donors in Tehran province 2005 - 2011. Arch Iran Med 2014; 17(9):613-20.

13. Doosti A, Amini-Bavil-Olyaee S, Tajbakhsh E, Adeli A, Mahboudi F. Prevalence of viral hepatitis and molecular analysis of HBV among voluntary blood donors in west Iran. New Microbiol 2009; 32(2):193-8.

14. Hashemi FB, Pourakbari B, Yazdi JZ. Frequency of chlamydia trachomatis in women with cervicitis in Tehran, Iran. Infect Dis Obstet Gynecol 2009; 2009:67014.

15. Ghazvini K, Ahmadnia H, Ghanaat J. Frequency of Chlamydia trachomatis among male patients with urethritis in northeast of Iran detected by polymerase chain reaction. Saudi J Kidney Dis Transpl 2012; 23(2):31620.

16. Bakhtiari A, Firoozjahi A. Chlamydia trachomatis infection in women attending health centres in Babol: prevalence and risk factors. East Mediterr Health J 2007; 13(5):1124-31.

17. Chamani-Tabriz L TM, Akhondi MM, MosaviJarrahi A, Zeraati H, Ghasemi J, Asgari S, Kokab A, Eley AR. Chlamydia trachomatis prevalence in Iranian women attending obstetrics and gynaecology clinics. Pak J Biol Sci 2007; 10(24):4490-4.

18. Ghanaat J, Afshari JT, Ghazvini K, Malvandi M. Prevalence of genital Chlamydia in Iranian males with urethritis attending clinics in Mashhad. East Mediterr Health J 2008; 14(6):1333-7.

19. Kassaian N, Ataei B, Yaran M, Babak A, Shoaei P, Ataie M. HIV and other sexually transmitted infections in women with illegal social behavior in Isfahan, Iran. Adv Biomed Res 2012; 1:5.

20. Yeganeh O, Jeddi-Tehrani M, Yaghmaie F, Kamali K, Heidari-Vala H, Zeraati H, et al. A survey on the prevalence of Chlamydia trachomatis and Mycoplasma genitalium infections in symptomatic and asymptomatic men referring to urology clinic of labbafinejad hospital, tehran, iran. Iran Red Crescent Med J 2013; 15(4):340-4.

21. Afrakhteh M, Beyhaghi H, Moradi A, Hosseini SJ, Mahdavi A, Giti S, et al. Sexually transmitted infections in Tehran. J Family Reprod Health 2008; 2(3):123-8.

22. Haghighi Hasanabad M, Mohammadzadeh M, Bahador A, Fazel N, Rakhshani H, Majnooni
A. Prevalence of Chlamydia trachomatis and Mycoplasma genitalium in pregnant women of Sabzevar-Iran. Iran J Microbiol 2011; 3(3):123-8.

23. Swiss.World Health Organization. Prevalence and incidence of selected sexually transmitted infections Chlamydia trachomatis, Neisseria gonorrhoeae, syphilis and Trichomonas vaginalis Methods and results used by WHO to generate 2005 estimates. Geneve: World Health Organization; 2011. Available from: http://apps.who.int/iris/bitstream/10665/44735/ 1/9789241502450_eng.pdf

24. Afrasiabi S, Moniri R, Samimi M, Khorshidi A, Mousavi SG. The prevalence of Endocervical Chlamydia trachomatis infection among young females in Kashan, Iran. Jundishapur J Microbiol 2015; 8(4):e15576.

25. Shahcheraghi F, Shafiei M, Valadkhani Z. Detection of Neisseria gonorrhoeae from vaginal swabs of Ewin, Rajaii shahr, Karaj and Varamin female prisoners by PCR and culture methods. Pak J Biol Sci 2010; 13(4):198-200.

26. Kazerooni PA, Motazedian N, Motamedifar M, Sayadi M, Sabet M, Lari MA, et al. The prevalence of human immunodeficiency virus and sexually transmitted infections among female sex workers in Shiraz, South of Iran: by respondent-driven sampling. Int J STD AIDS 2014; 25(2):155-61.

27. Hassanzadeh P, Maradaneh J, Motamedifar M. Conventional agar-based culture method, and nucleic acid amplification test (NAAT) of the cppB gene for detection of neisseria gonorrhea in pregnant women endocervical swab specimens. Iran Red Crescent Med J 2013; 15(3):207-11.

28. Mohammadali F, Pourfathollah AA. Changes in frequency of $\mathrm{HBV}, \mathrm{HCV}, \mathrm{HIV}$ and syphilis infections among blood donors in Tehran province 2005 - 2011. Arch Iran Med 2014; 17(9):613-20.

29. Khedmat H, Fallahian F, Abolghasemi H, Alavian SM, Hajibeigi B, Miri SM, et al. Seroepidemiologic study of hepatitis B virus, hepatitis C virus, human immunodeficiency virus and syphilis infections in Iranian blood donors. Pak J Biol Sci 2007; 10(24):4461-6.

30. Navadeh S, Mirzazadeh A, Mousavi L, Haghdoost A, Fahimfar N, Sedaghat A. HIV, HSV2 and Syphilis prevalence in female sex workers in Kerman, South-East Iran; using respondent-driven sampling. Iran J Public Health 2012; 41(12):60-5.

31. Nokhodian Z, Yazdani M, Yaran M, Shoaei P, Mirian M, Ataei B, et al. Prevalence and risk factors of HIV, Syphilis, Hepatitis B and C 
among female prisoners in Isfahan, Iran. Hepat Mon 2012; 12(7):442-7.

32. Haghgoo SM, Joula H, Mohammadzadeh R, Sabour S, Yousefi R, Ghahramani G, et al. Epidemiology of HIV/AIDS in the East Azerbaijan province, northwest of Iran. Jundishapur J Microbiol 2015; 8(8):e19766.

33. Murray CJ, Ortblad KF, Guinovart C, Lim SS, Wolock TM, Roberts DA, et al. Global, regional, and national incidence and mortality for HIV, tuberculosis, and malaria during 1990-2013: a systematic analysis for the Global Burden of Disease study 2013. Lancet 2014; 384(9947):1005-70.

34. United Nations Programme on HIV/AIDSUNAIDS. Second Independent Evaluation of UNAIDS. Paper Presented at: $25^{\text {th }}$ Meeting of the UNAIDS Programme Coordinating Board; 2009 December 8-10; Switzerland, Geneva. No.:UNAIDS/PCB(25)/09.CRP.18.

35. Malekinejad M, Mohraz M, Razani N, Akbari G, McFarland W, Khairandish P, et al. High HIV prevalence in a respondent-driven sampling survey of injection drug users in Tehran, Iran. AIDS Behav 2015; 19(3):440-9.

36. Khajehkazemi R, Osooli M, Sajadi L, Karamouzian M, Sedaghat A, Fahimfar N, et al. HIV prevalence and risk behaviours among people who inject drugs in Iran: the 2010 National Surveillance Survey. Sex Transm Infect 2013; 89(Suppl 3): iii29-32.

37. Iranian national center for AIDS prevention (NcoApco). statistics and information [Internet] 2017 Mar. Available from: http://aids.ir/Info

38. Haghdoost AA, Mostafavi E, Mirzazadeh A,
Navadeh S, Feizzadeh A, Fahimfar N, et al. Modelling of HIV/AIDS in Iran up to 2014. J AIDS HIV Res 2011; 3(12):231-9.

39. WHO. Global Health Observatory country views. Iran (Islamic Republic of) statistics summary (2002 - present). Geneve: World Health Organization; 2016 Sep. Available from:

http://apps.who.int/gho/data/node.country.coun try-IRN?lang=en

40. Swiss. World Health Organization. Wourld health statistics 2015. Geneve: World Health Organization; 2015. Available from: http://apps.who.int/iris/bitstream/10665/17025 0/1/9789240694439_eng.pdf?ua=1

41. Karamouzian M, Nasirian M, Sedaghat A, Haghdoost AA. HIV in Iran. Lancet 2014; 383(9922): 1040.

42. Iranian national center for AIDS prevention (NcoApco). Center for Contagious Disease Management. positive clubs; [Internet] 2017 Mar. Available from: http://aids.behdasht.gov.ir/index.aspx?fkeyid= \&siteid=328\&pageid $=50520$.

43. Nourabadi GhR. Youth Empowerment on HIV/AIDS Prevention. Geneve: UNAIDS, Programme Coordinating Board; 2013 Dec. Available from: http://www.unaids.org/sites/default/files/en/me $\mathrm{dia} /$ unaids/contentassets/documents/pcb/2013/p cb33/agendaitems/presentations/Item9_Iran_Y outhEmpowerment.pdf.

44. Noroozi M, Taleghani F, Merghati-khoei, ES, Tavakoli M, Gholami A. Premarital sexual relationships: Explanation of the actions and functions of family. Iran J Nurs Midwifery Res 2014; 19(4):424-31. 